\title{
Special issue: mathematical pharmacology
}

\author{
Wojciech Krzyzanski ${ }^{1}$ (D) J. G. Coen van Hasselt ${ }^{2}$
}

Received: 27 December 2017 / Accepted: 29 December 2017 / Published online: 3 January 2018

(C) Springer Science+Business Media, LLC, part of Springer Nature 2018

Pharmacology as a biomedical science has not yet received the attention of applied mathematicians such as has been the case for the field of mathematical biology. In recognition of increasing relevance of mathematical ideas and methods in the realm of pharmacology van der Graaf et al. coined the term Mathematical Pharmacology for the field of study that is aimed at using mathematical approaches to achieve a better understanding of pharmacological processes [1]. Although examples of tools applicable for mathematical pharmacology have been limited to deterministic dynamical systems, a much broader arsenal of techniques is available. More importantly, many of these methods have been successfully applied in biomedical sciences and they continue to attract more users. The purpose of this special issue of Journal of Pharmacokinetics and Pharmacodynamics is to increase awareness of modelers of currently applied mathematical techniques and to popularize Mathematical Pharmacology as a discipline of mathematical biology. We focused on both the application of mathematical approaches as well as overlap with advanced statistical methods. To this aim, we invited experts in their fields to contribute to this special issue a manuscript that would briefly introduce a mathematical technique of interest and provide examples of applications to pharmacological problems.

Mathematical techniques presented in this special issue have been organized according to their level of mathematical complexity rather than therapeutic areas or similarity of pharmacological systems they describe. Classical methods of dynamical systems analysis are followed by delay differential equations and distributed delay differential equations with examples of bifurcation analysis. Problems arising in

Wojciech Krzyzanski

wk@buffalo.edu

1 Department of Pharmaceutical Sciences, University at Buffalo, Buffalo, NY, USA

2 Division of Systems Biomedicine and Pharmacology, Leiden Academic Centre for Drug Research, Leiden University, Leiden, The Netherlands estimation of model parameters are introduced in the context of structural identifiability analysis. Numerical approaches to alternative methods of solving ordinary differential equations are represented by the inductive linearization technique. Methods of fractional calculus are explained using examples of fractional differential equations. Markov processes and stochastic differential equations are applied in mixed effects hidden Markov models. Optimal control theory demonstrates utility of calculus of variations. Techniques of model reduction such as proper lumping and input response index are applied to systems pharmacology models. The special issue concludes with a review of Boolean networks techniques of modeling signaling pathways in complex pharmacological systems. Although majority of articles are original research publications, several contributions to this special issue are written in a format of review.

By no means selection of presented topics is comprehensive, but rather merely representative of a broader spectrum of problems specific to mathematical pharmacology that can be solved by existing mathematical techniques and inspire development of new ones. Examples of methods that have not been included are chaotic dynamical systems [2] and partial differential equations [3]. We hope that future issues of Journal of Pharmacokinetics and Pharmacodynamics will contain growing number of contributions falling into the scope of mathematical pharmacology.

Acknowledgements We would like to thank 43 reviewers, particularly those of mathematical background, for their constructive comments which help us to uphold mathematical rigor in all articles of this special issue.

\section{References}

1. van der Graaf PH, Benson N, Peletier LA (2016) Topics in mathematical pharmacology. J Dyn Diff Equat 28:1337-1356

2. Dokoumetzidis A, Iliadis A, Macheras P (2001) Nonlinear dynamics and chaos theory: concepts and applications relevant to pharmacodynamics. Pharm Res 18(4):415-426

3. Krzyzanski W (2015) Pharmacodynamic models of age-structured cell populations. J Pharmacokinet Pharmacodyn 42:573-589 